University of Nebraska - Lincoln

DigitalCommons@University of Nebraska - Lincoln

Norman R. Simon Papers

Research Papers in Physics and Astronomy

$11-1985$

\title{
CLASSICAL CEPHEID LIGHT CURVES REVISITED
}

Norman R. Simon

University of Nebraska - Lincoln, nsimon@unl.edu

Thomas J. Moffett

Purdue University, moffett@physics.purdue.edu

Follow this and additional works at: https://digitalcommons.unl.edu/physicssimon

Simon, Norman R. and Moffett, Thomas J., "CLASSICAL CEPHEID LIGHT CURVES REVISITED" (1985).

Norman R. Simon Papers. 56.

https://digitalcommons.unl.edu/physicssimon/56

This Article is brought to you for free and open access by the Research Papers in Physics and Astronomy at DigitalCommons@University of Nebraska - Lincoln. It has been accepted for inclusion in Norman R. Simon Papers by an authorized administrator of DigitalCommons@University of Nebraska - Lincoln. 


\title{
CLASSICAL CEPHEID LIGHT CURVES REVISITED
}

\author{
NORMAN R. SIMON \\ Department of Physics and Astronomy, University of Nebraska, Lincoln, Nebraska 68588-0111 \\ AND \\ THOMAS J. MOFFETT \\ Department of Physics, Purdue University, West Lafayette, Indiana 47907 \\ Received 1985 July 7, revised 1985 August 31
}

\begin{abstract}
Fourier decompositions are made of the $B, V, R$, and $I$ classical Cepheid observations of Moffett and Barnes. The Fourier diagrams for $V$ agree very well with the corresponding plots of Simon and Lee. The $B, R$, and $I$ diagrams resemble the plots for $V$, but with small, systematic shifts in the phases $\phi_{21}$ and $\phi_{31}$. The 2.5-day star, DT Cyg, is confirmed as a likely overtone pulsator by its anomalous position in the Fourier plots. Finally, we study the Fourier phase quantity $\phi_{41}$, and introduce phase-phase diagrams to look at the Hertzsprung progression. Discontinuities in these diagrams support the idea that the long-period and short-period Cepheids may reach their limit cycles in different ways.
\end{abstract}

Key words: stars: Cepheids-stars: pulsation

\section{Introduction}

The variations of pulsating stars can be treated quantitatively by the simple but useful technique of Fourier decomposition. References to early applications of this method may be found in Payne-Gaposchkin (1947). More recently Simon and Lee (1981) independently reformulated this technique and applied it to the light curves of classical Cepheids. It was found that certain phase differences and amplitude ratios among the Fourier terms displayed regular progressions with period, corresponding to the Hertzsprung sequence.

Subsequently, the same framework was used to analyze the velocity curves of classical Cepheids (Simon and Teays 1983), the light curves of RR Lyrae stars in the field (Simon and Teays 1982) and in the globular cluster $\omega$ Centauri (Petersen 1984), and to treat hydrodynamic models of the variations of BL Herculis stars (Hodson, Cox, and King 1982). Comparison of theory and observations using the Fourier technique have been made by Simon and Davis (1983), and Simon $(1984 a, b)$. In addition, Gieren (1982) emphasized the utility of this method as a pulsational mode discriminator, while Antonello and Mantegazza (1984) applied it to double-mode Cepheids including the unique star CO Aurigae.

In view of such widespread use of the Fourier framework, as well as its potential employment in further studies, we have decided to reexamine and enlarge the original application to the classical Cepheids. The impetus for this investigation comes from the availability of new observations-namely, the large, and homogeneous set of photometric data obtained by Moffett and Barnes (1980, 1984).

In what follows, we shall verify the Fourier plots of Simon and Lee (1981), extend the analysis to wavelengths other than the visual and to periods longer than 17 days, and investigate additional properties of the Fourier decompositions, not studied in previous work. In Section II we discuss Fourier decomposition of $V$-magnitude data, while the $B, R$, and $I$ observations are treated in Section III. Color-color comparisons are made in Section IV. Finally, in Section V we study the Fourier phase difference, $\phi_{41}$, and introduce phase-phase (as opposed to phase-period) plots for the Fourier coefficients.

\section{V-Magnitude Observations}

Fourier decomposition was performed on the $V$-magnitude data of Moffett and Barnes (1980, 1984). We have adopted the notation for the Fourier parameters as used by Simon and Lee (1981). The photometric data were fitted with a Fourier series of the form

$$
A_{0}+\sum_{i=1}^{i_{\max }} A_{i} \cos \left[i \omega\left(t-t_{0}\right)+\phi_{i}\right]
$$

where, for a given fit, the index $i$ runs from 1 to $2 \leq i_{\max } \leq$ 8 . The amplitude ratios, $R_{i j}$, are defined by equation (2)

$$
R_{i j}=A_{i} / A_{j}
$$

The Fourier phase differences are defined by equation (3)

$$
\phi_{i j}=\phi_{i}-i \phi_{j} .
$$

The values of the various Fourier coefficients for the $V$-magnitude fits are given in Moffett and Barnes (1985).

Figure 1 shows a plot of $\phi_{21}$ vs. period for an unedited sample of the data. This diagram closely resembles that of Simon and Lee (1981), except that there is more scatter, particularly among the stars of shortest period. We have edited the data by removing the stars listed below which were suspect for the stated reasons. We did not remove any stars solely on the basis of their position in the Fourier plots. 


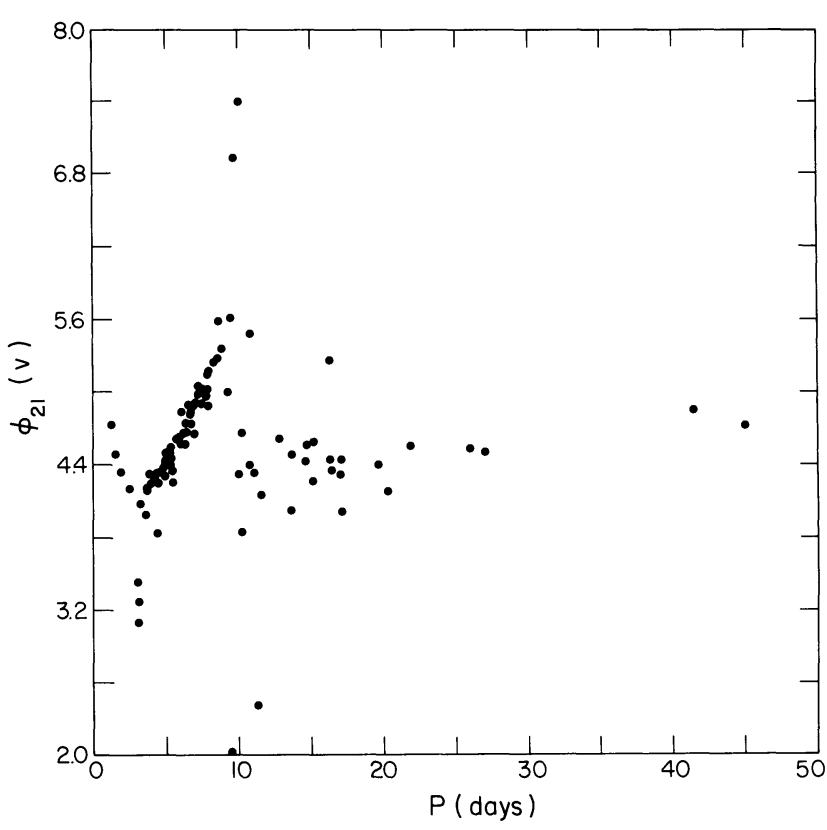

FIG. 1-The phase difference $\phi_{21}=\phi_{2}-2 \phi_{1}$ versus period for the $V$-magnitude in the unedited sample.

BL Herculis and SW Tauri: These are type II Cepheids, as is well known.

EV Scuti, DX Geminorum, SZ Tauri, and V532 Cygni: Due to the low amplitudes of these stars, the second-order Fourier terms were not well enough determined by the present observations. However, we do not dismiss the possibility that some or all of the discrepant points represented by these objects are real. Further investigation of these stars will be necessary.

SS Scuti: Fourier decomposition (Simon 1984a) of the considerably larger data set of Gieren (1981) gives a different value of $\phi_{21}$, one that is completely normal for the period of this star.

FF Aquilae and V924 Cygni: These stars have very low amplitudes (see remarks above). In addition, FF Aql is a binary. More study will be necessary.

FN Aquilae: This star also has a low amplitude and an extremely small second-order Fourier term. However, it seems normal for its period, resembling stars like DD Cassiopeiae and $\zeta$ Geminorum.

$A L$ Virginis: 'This is a type II Cepheid.

CS Monocerotis, AA Geminorum, and SZ Monocerotis: The phase coverage in these cases was too poor to accurately determine the Fourier coefficients.

In Figure 2 we present a plot of $\phi_{21}$ vs. period for the edited sample. The diagram now has a cleaner appearance and illustrates the remarkable homegeneity of the type I Cepheids. The two short-period stars which depart from the sequence are SU Cassiopeiae and DT Cygni. The former star is a suspected overtone pulsator (Gieren 1982), and Figure 2 leads us to suggest that DT Cyg is also a strong candidate for overtone pulsation. A similar con-

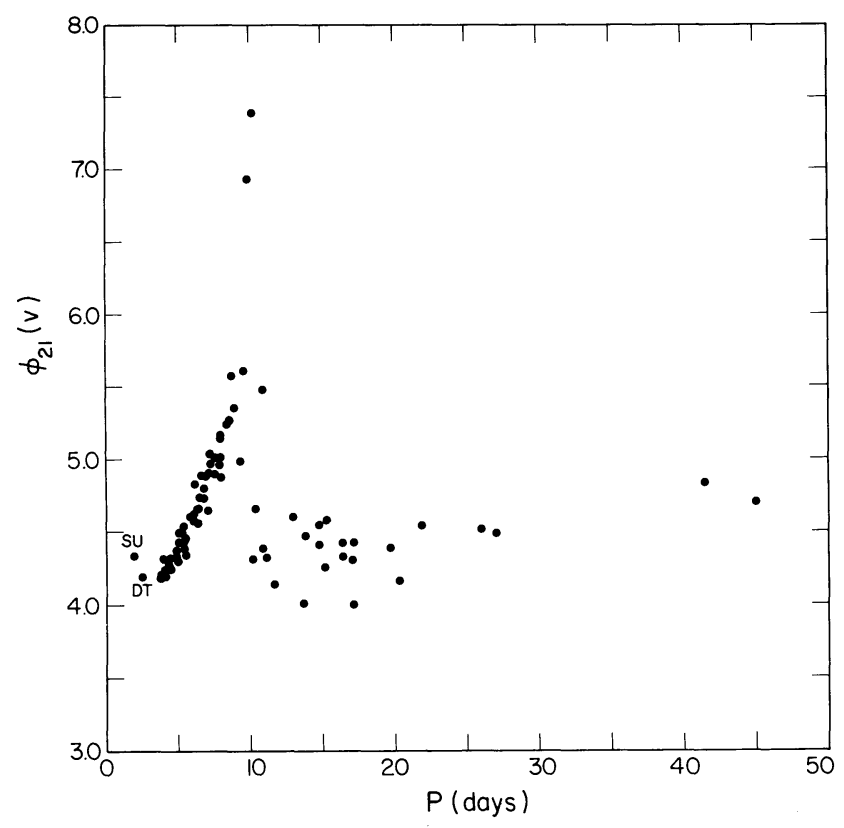

FIG. 2-The phase difference $\phi_{21}(V)$ versus period for the edited $V$ magnitude sample.

clusion regarding this star was reached by Arellano-Ferro (1984) on different grounds. A third star, AZ Cen, which is absent from the present sample, also has an anomalous position on the Fourier diagrams (Simon 1984a), indicating that it, too, may be an overtone pulsator.

Figures 3 and 4 , respectively, display the amplitude ratio $R_{21}$ and the phase difference $\phi_{31}$ for the edited sample. These plots are also very similar to the corresponding diagrams in Simon and Lee (1981). The points representing SU Cas and DT Cyg stand out at the lower left of Figure 3. In Figure 4, SU Cas is again easily distinguished but DT Cyg has not been plotted because its third-order Fourier term is vanishingly small. We note that the Moffett-Barnes sample extends to quite long periods, 40 days, considerably further than the stars treated in the SimonLee study. Interestingly enough, the trend shown by the middle-period stars seems to extend to the longest periods in all three diagrams, Figures 2-4. However, one should be cautious about this conclusion since the longperiod sample is very small and includes some stars with poor phase coverage.

\section{III. $B$-, R-, and I-Magnitude Observations}

The question of how the light curves of pulsating stars change with wavelength is an interesting one to explore in detail. While the work of Simon and Lee (1981) provided a $V$-magnitude baseline against which future observations (e.g., of extragalactic Cepheids) may be compared, a similar framework for other wavelengths has been lacking. This lack hampered a study by Teays and Simon (1982) of published $B$-magnitude variations of Cepheids in M31. In addition, because modern panoramic detec- 


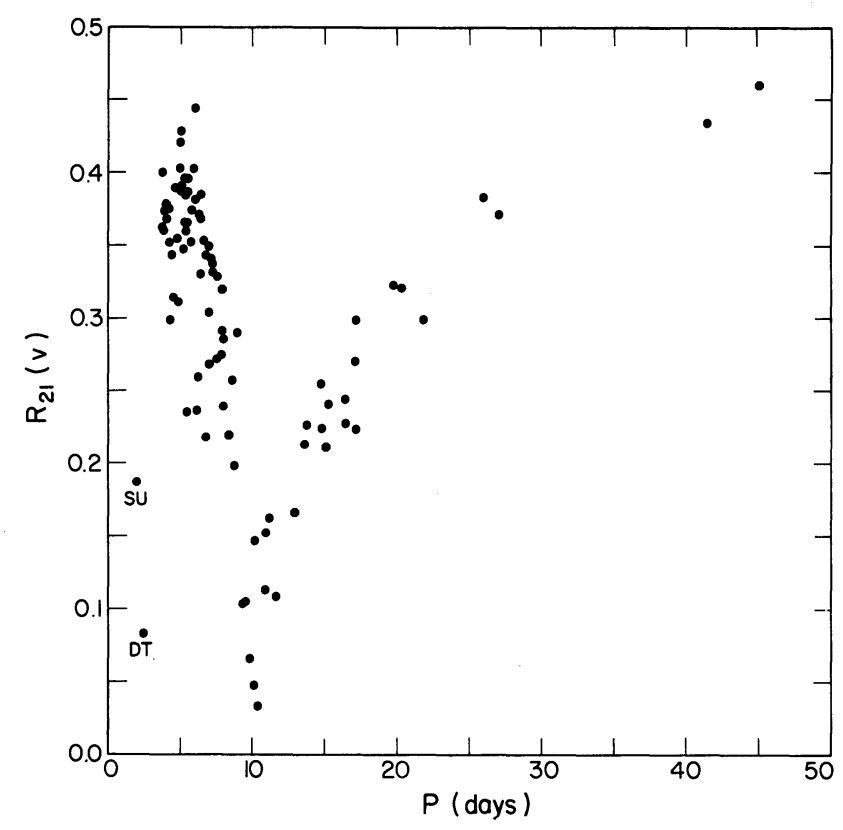

FIG. 3-The amplitude ratio $R_{21}=A_{2} / A_{1}$, versus period for $V$-magnitude data.

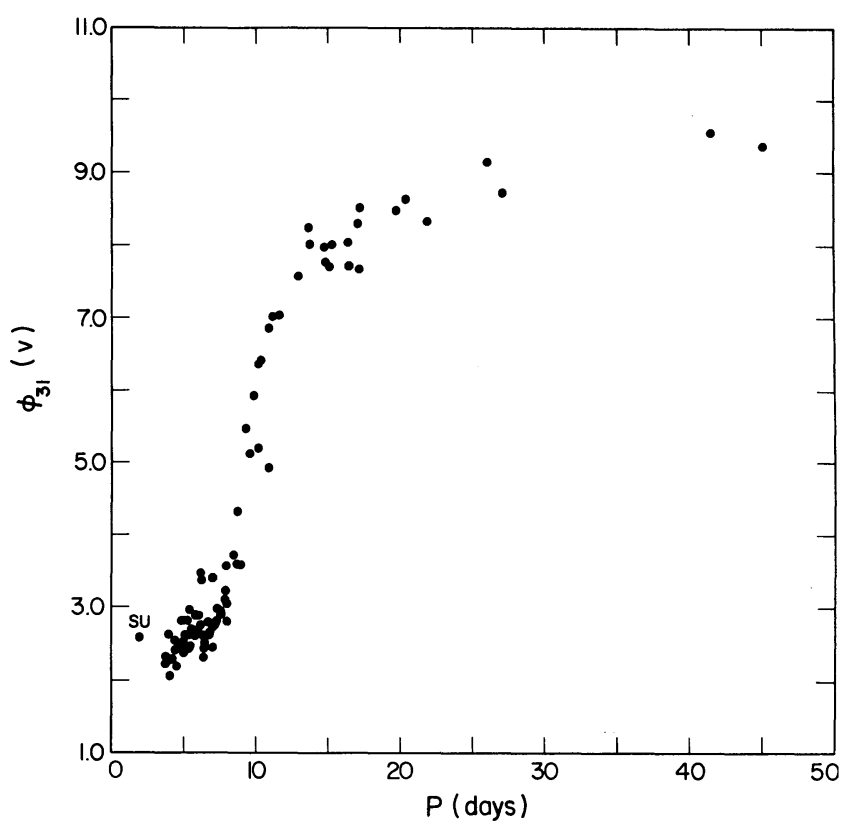

FIG. 4-The phase difference $\phi_{31}=\phi_{3}-3 \phi_{1}$ versus period for $V$-magnitude data.

tors are much more sensitive at longer wavelengths, baseline data in $R$ and $I$ would also be very desirable. The large Moffett-Barnes sample offers a unique opportunity to obtain such data.

Figures 5-7 show $\phi_{21}, R_{21}$, and $\phi_{31}$, respectively, for the $B$-magnitude observations. The sample is the same as the edited sample above. The $B$-plots closely resemble those for $V$, except that the former points are systematically shifted to slightly smaller values of $\phi_{21}$ and $\phi_{31}$. We shall treat this property in more detail later.

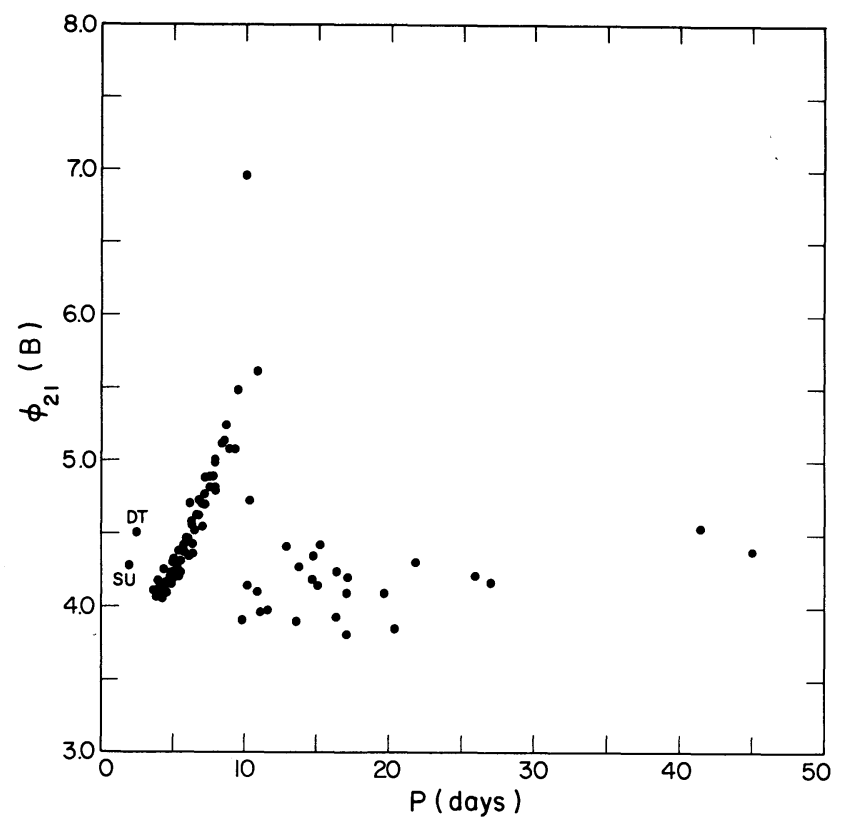

FIG. 5-The phase difference $\phi_{21}$ versus period for $B$-magnitude data.

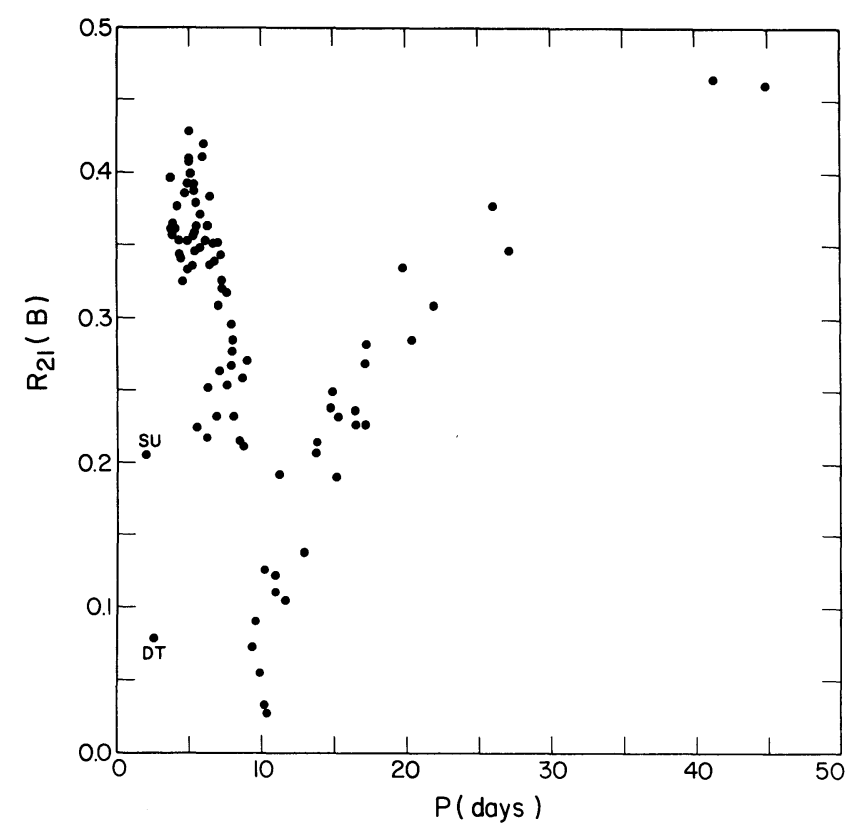

FIG. 6-The amplitude ratio $R_{21}$ versus period for $B$-magnitude data.

Turning to the $R$-magnitude observations, we have plotted $\phi_{21}, R_{21}$, and $\phi_{31}$ in Figures 8-10. The sample here is somewhat smaller than that for $B$ or $V$ due to the well-known fall of amplitude with increasing wavelength. We have omitted those stars whose second and/or third Fourier components have become too small for accurate determination. This occurs when the size of a given component is less than the standard deviation of the entire fit. The Fourier plots for $R$ are again very similar to those for $V$ except that the $R$ points are shifted toward slightly higher values of $\phi_{21}$ and $\phi_{31}$. Once again, SU Cas stands 


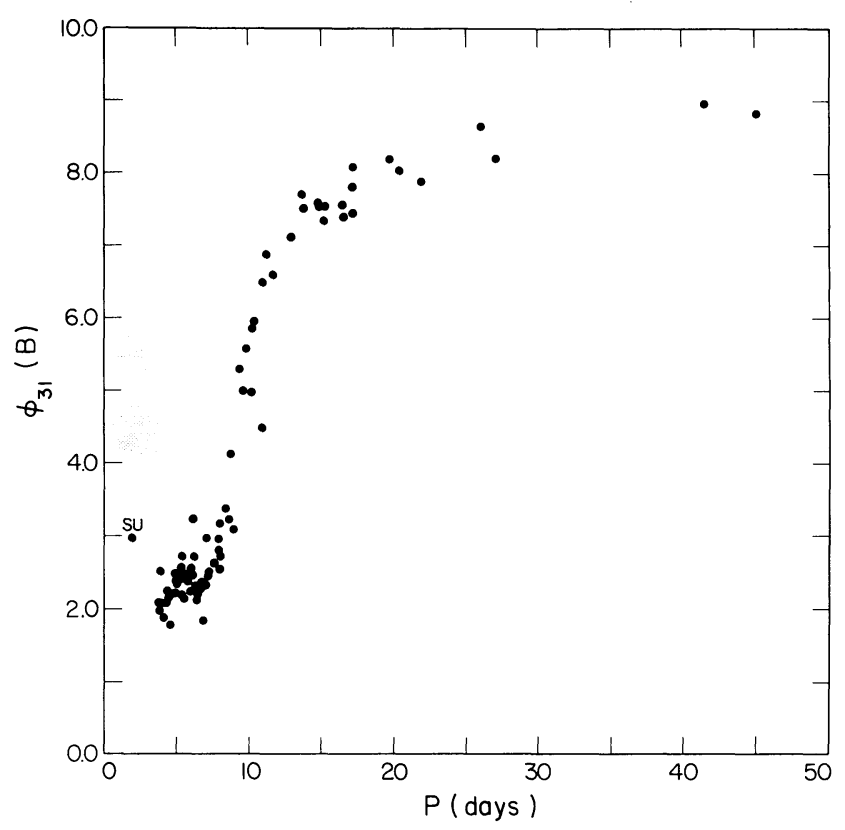

FIG. 7-The phase difference $\phi_{31}$ versus period for the $B$-magnitude data.

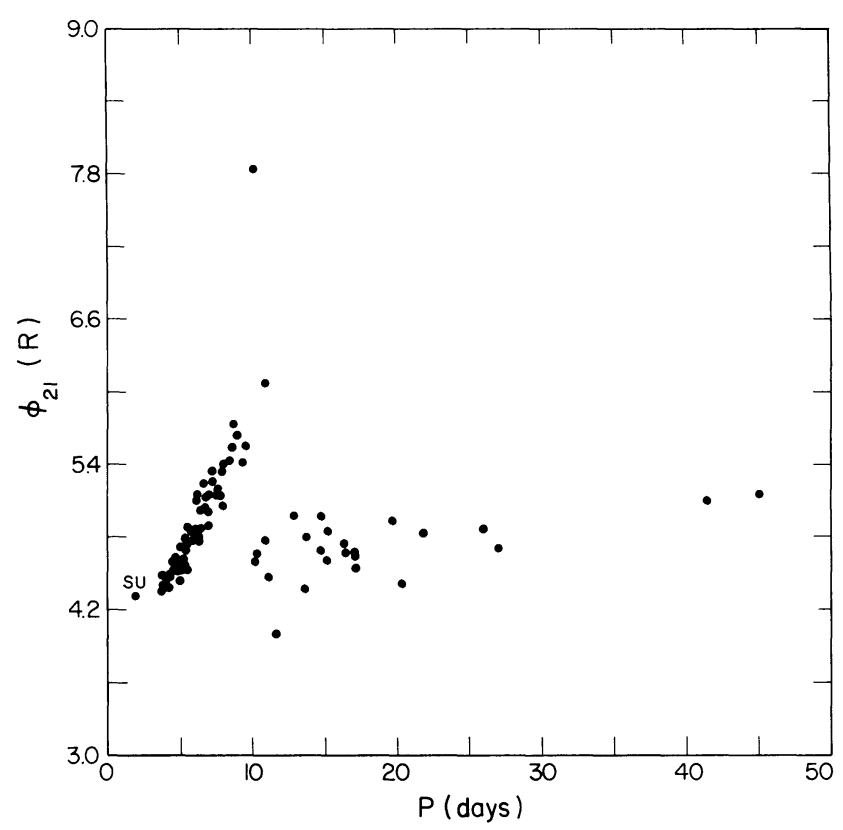

FIG. 8 -The phase difference $\phi_{21}$ versus period for the $R$-magnitude data.

out in all three diagrams. The other suspected overtone pulsator, DT Cyg, is not plotted here because its second and third Fourier terms are very small in $R$.

Fourier decompositions are given for the $I$-magnitude observations in Figures 11-13 which display $\phi_{21}, R_{21}$, and $\phi_{31}$, respectively. Again, a number of stars have been omitted due to their low amplitudes. Although the Fourier diagrams show more scatter here than at shorter wavelengths (an effect attributable to the reduced ampli-

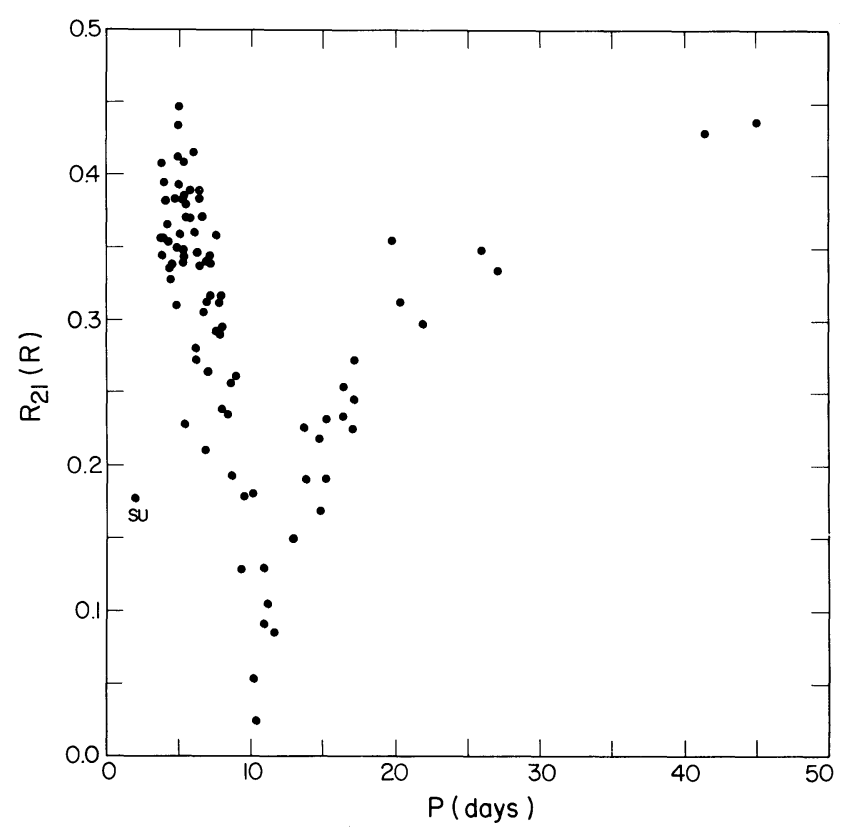

FIG. 9-The amplitude ratio $R_{21}$ versus period for the $R$-magnitude data.

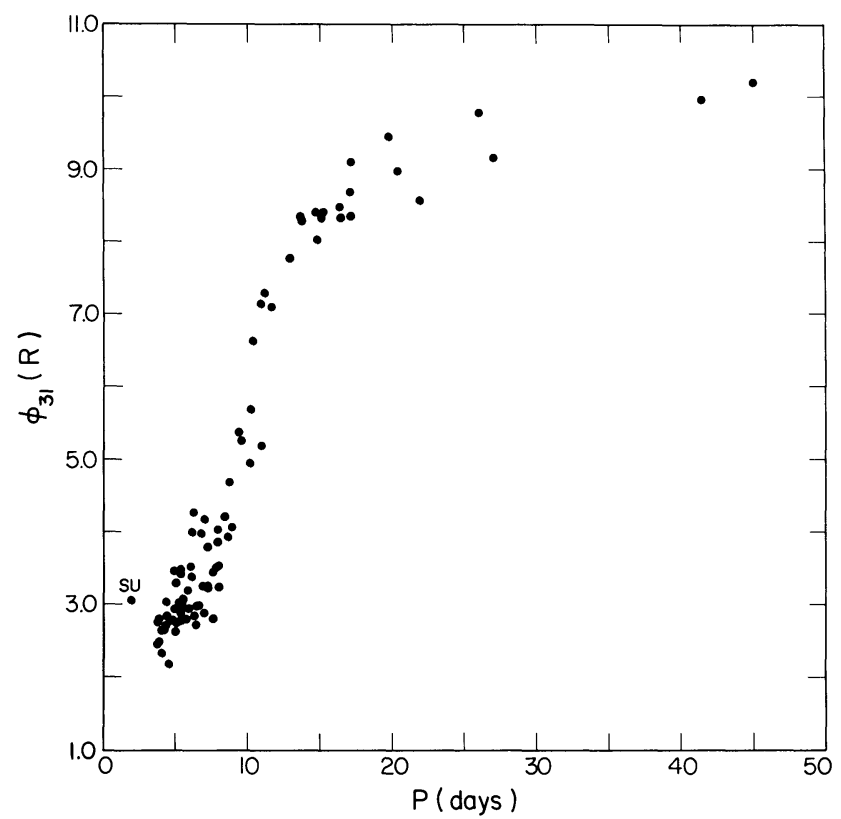

FIG. 10 -The phase difference $\phi_{31}$ versus period for the $R$-magnitude data.

tudes noted above), the resemblance of the $I$-magnitude plots to those for the other colors is clear. However, the values of $\phi_{21}$ and $\phi_{31}$ are systematically larger for $I$ than they were for $R$.

\section{Color-Color Comparisons}

In the previous section we have seen a trend of increasing values of $\phi_{21}$ and $\phi_{31}$ with increasing wavelength of the observations. Table I documents this trend by displaying 


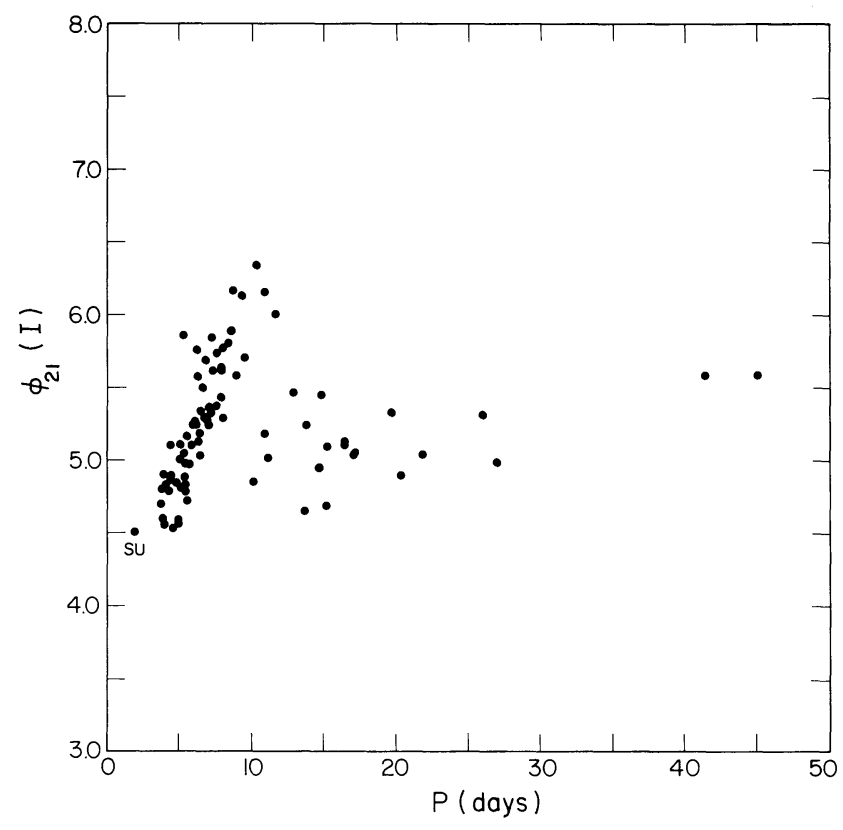

FIG. 11-The phase difference $\phi_{21}$ versus period for the $I$-magnitude data.

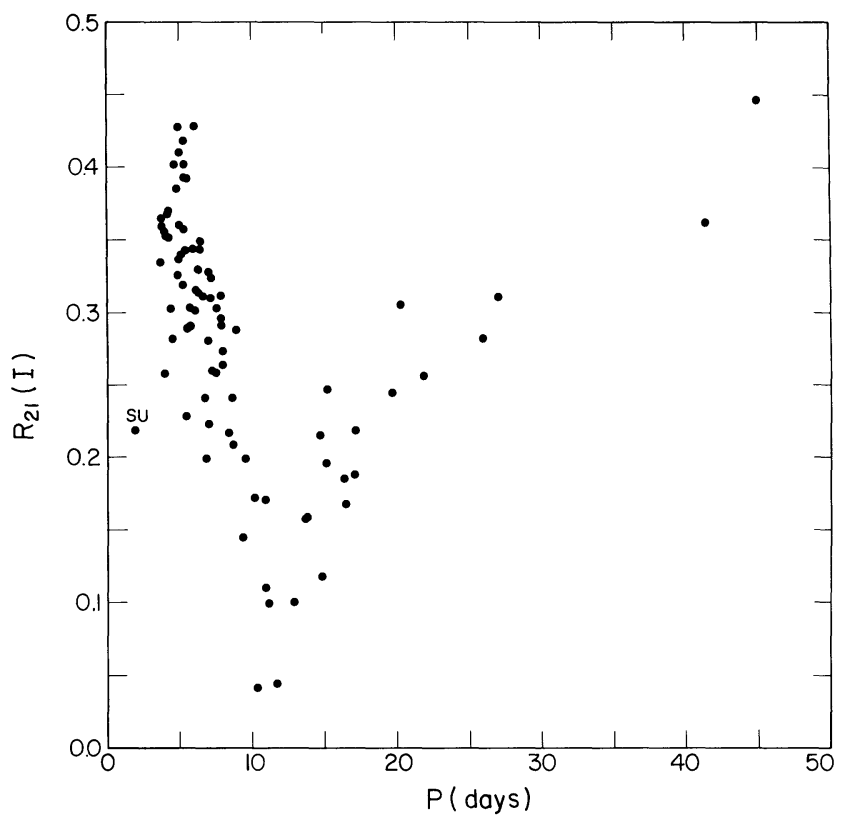

FIG. 12-The amplitude ratio $R_{21}$ versus period for the $I$-magnitude data.

average values of the color-color differences for the quantities $\phi_{21}$ and $\phi_{31}$ in $B$ and $V$ and in $R$ and $V$. For convenience, the period axis has been broken into three segments: $3.8<P<5$ d $1,5.1<P<10$. 9 , and $10.9<P<45^{\mathrm{d}}$. Formal errors are also given. It is clear from Table $I$ that $\phi_{21}$ and $\phi_{31}$ do indeed increase from $B$ to $V$ to $R$, and that this change is larger for the stars of longer period. Typical differences are $\left\langle\phi_{21}(B)-\phi_{21}(V)\right\rangle \sim-0.20,\left\langle\phi_{21}(R)-\right.$ $\left.\phi_{21}(V)\right\rangle \sim 0.25$, and $\left\langle\phi_{31}(B)-\phi_{31}(V)\right\rangle \sim-0.30,\left\langle\phi_{31}(R)\right.$ $\left.-\phi_{31}(V)\right\rangle \sim 0.40$. These results are similar to

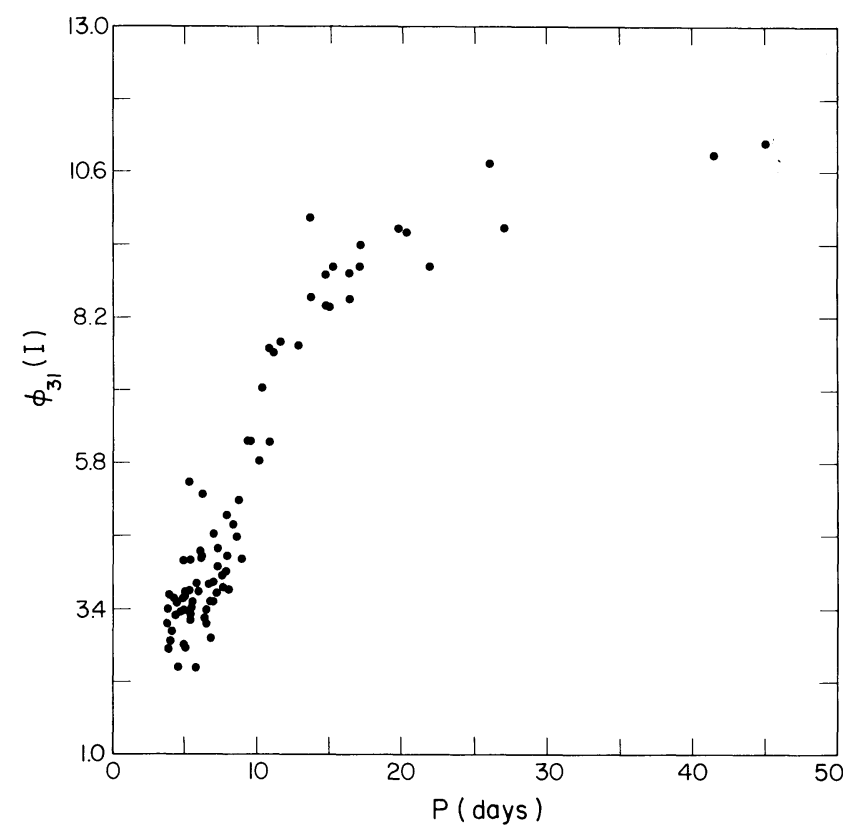

FIG. 13-The phase difference $\phi_{31}$ versus period for the $I$-magnitude data.

those found by Petersen and Hansen (1984) for a handful of type II pulsators.

We complete our discussion of color-color variations by considering a number of Fourier decompositions made for the bolometric magnitude, $m_{\text {bol }}$. Table II shows how $\mathrm{R}_{21}, \phi_{21}$, and $\phi_{31}$ changes as one goes from $V$-magnitude to bolometric magnitude data. Bolometric magnitudes were determined for the eight stars listed by adding a bolometric correction to each $V$-magnitude observation according to the scheme of Schmidt (1985). The bolometric light curves were then Fourier decomposed in the usual manner. The differences entered in Table II are in the form $\Delta \equiv m_{\text {bol }}-V$ (e.g., $\left.\Delta R_{21}=R_{21}\left(m_{\text {bol }}\right)-R_{21}(V)\right)$. As expected, the Fourier quantities for $m_{\text {bol }}$ are not too different from those for $V$. We may thus compare a series of hydrodynamic light curves with $V$-magnitude observations without significant error. However, it is obvious from Table II that some caution is necessary in this regard when one is attempting to model a particular star.

\section{The Fourier Quantity $\phi_{41}$}

Petersen (1984) and Petersen and Hansen (1984) included higher-order Fourier terms in their discussions of type II pulsators. Indeed, in a preliminary study of the short-period type II Cepheids, Simon (1984b) found that a suspected resonance in the period spectrum of these stars showed up much more strongly in $\phi_{31}$ and $\phi_{41}$ than it did in $\phi_{21}$. Because the Fourier phase difference $\phi_{41} \equiv \phi_{4}$ $-4 \phi_{1}$ (e.g., Petersen and Hansen 1984) may play a useful role in our attempt to understand the type II stars and since the original Simon-Lee study of the type I stars did not include this quantity, we have decided to use the 
TABLE I

Average Color - Color Differences in $\phi_{21}$ and $\phi_{31}$

\begin{tabular}{|c|c|c|c|c|}
\hline Period & $\left\langle\phi_{2 I}(\mathrm{~B})-\phi_{2 I}(\mathrm{~V})\right\rangle$ & $\left\langle\phi_{2 I}(\mathrm{R})-\phi_{2 I}(\mathrm{~V})\right\rangle$ & $\left.<\phi_{31}(\mathrm{~B})-\phi_{31}(\mathrm{~V})\right\rangle$ & $<\phi_{31}(\mathrm{R})-\phi_{31}(\mathrm{~V})>$ \\
\hline (days) & $\pm \delta$ (no. of points) & $\pm \delta \quad$ (no. of points) & $\pm \delta$ (no. of points) & $\pm \delta$ (no. of points) \\
\hline \multirow[t]{2}{*}{$3.8-5.1$} & -0.143 & 0.204 & -0.246 & 0.342 \\
\hline & \pm 0.029 (16) & \pm 0.055 (17) & \pm 0.078 & \pm 0.088 (15) \\
\hline \multirow[t]{2}{*}{$5.1-10.9$} & -0.170 & 0.239 & -0.299 & 0.428 \\
\hline & \pm 0.068 & \pm 0.062 & \pm 0.111 (39) & \pm 0.158 (38) \\
\hline \multirow[t]{2}{*}{$11.1-45$} & -0.239 & 0.311 & -0.449 & 0.415 \\
\hline & $\pm 0.072(20)$ & \pm 0.067 & $\pm 0.109(20)$ & \pm 0.190 \\
\hline
\end{tabular}

TABLE II

Differences Between Bolometric and V-Light Curves for Selected Stars $\Delta \equiv \mathrm{m}_{\mathrm{bol}}-\mathrm{V}$

$\begin{array}{lcccc}\text { STAR } & \begin{array}{c}\text { PERIOD } \\ \text { (days) }\end{array} & \Delta \mathrm{R}_{21} & \Delta \phi_{21} & \Delta \phi_{31} \\ \text { RT AUR } & 3.73 & 0.0004 & -0.0443 & -0.0788 \\ \text { T VUL } & 4.44 & 0.0001 & -0.0898 & -0.1414 \\ \text { UY PER } & 5.37 & 0.0048 & -0.1248 & -0.1214 \\ \text { U SGR } & 6.75 & -0.0011 & -0.1054 & -0.1646 \\ \text { DL CAS } & 8.00 & 0.0006 & -0.0894 & -0.1496 \\ \text { RX AUR } & 11.62 & -0.0017 & -0.1109 & -0.2613 \\ \text { CD CYG } & 17.07 & 0.0010 & -0.1339 & -0.2977 \\ \text { SV VUL } & 45.00 & 0.0072 & -0.2098 & -0.3496\end{array}$

Moffett-Barnes sample to investigate $\phi_{41}$ for the classical Cepheids.

Figure 14 shows a plot of $\phi_{41}$ vs. period for the $V$-magnitude observations. The sample of stars is reduced from that of Figures 2-4, since the fourth-order Fourier term was not well determined for stars of lower amplitude. The resonance sequence (Simon and Lee 1981) with its sharp break near ten days appears strikingly in $\phi_{41}$ whose progression qualitatively resembles that of $\phi_{21}$ (Fig. 2). However, one notes from Figure 14 a gap in $\phi_{41}$ between approximately $\pi / 2$ and $3 \pi / 2$. Because this void occurs in a well-populated region of the period axis (i.e., five to seven days), we suspect that it may be real. Unfortunately, at the moment, we have no explanation to offer for its presence.

The strong indication of the classical Cepheid resonance, $P_{2} / P_{0} \sim 0.5$, in the Fourier phase vs. period diagrams is aided by the fact that the loci, in the H-R diagram, of constant period and those of constant-period ratio, $P_{2} / P_{0}$, coincide for the type $\mathrm{I}$ stars. On the other

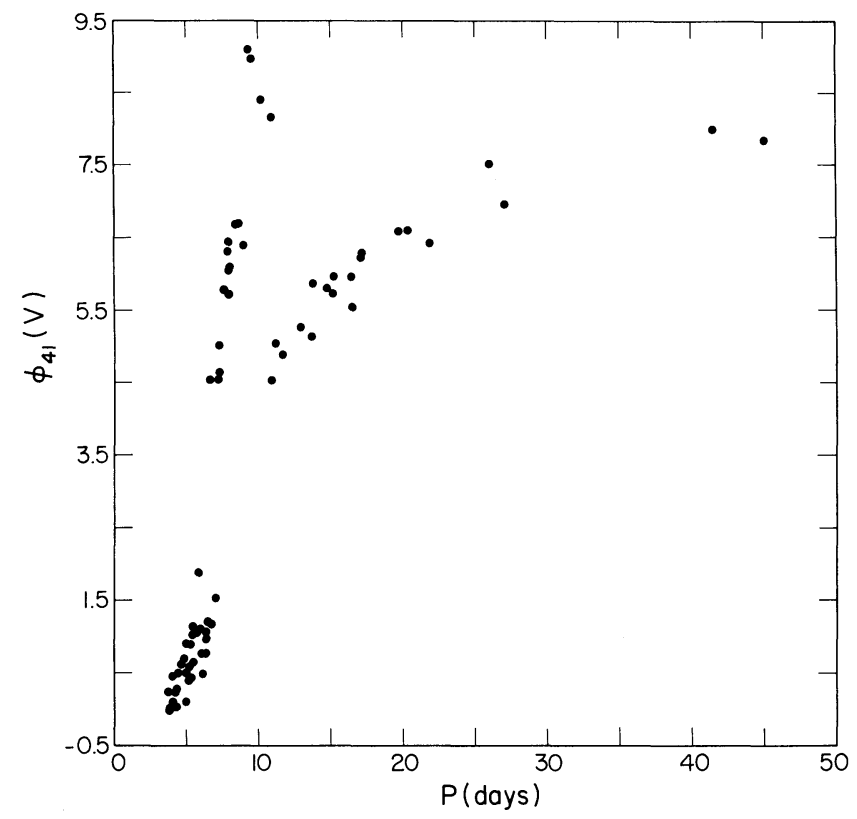

FIG. 14-The phase difference $\phi_{41}=\phi_{4}-4 \phi_{1}$ versus period for $V$-magnitude data.

hand, Hodson et al. (1982) have pointed out that these two loci diverge considerably for the short-period type II pulsators where the same resonance $\left(P_{2} / P_{0} \sim 0.5\right)$ is expected to play an important role. Because the resonance properties of the light curve depend on period-ratio, it would appear that the period itself is not a "good" variable for describing a resonance progression in these stars.

In some work now in progress, one of us (N.R.S.) has attempted to remedy this defect by plotting phase-phase diagrams, as opposed to phase-period diagrams, for the type II Cepheids. The results of this research will be reported elsewhere. However, in the present study we 
wish to establish some framework for the phase-phase plots by displaying them for the classical Cepheids. Figures 15-17 show diagrams of $\phi_{31}$ vs. $\phi_{21}, \phi_{41}$ vs. $\phi_{21}$, and $\phi_{41}$ vs. $\phi_{31}$, respectively, for the $V$-magnitude data. Because it is sometimes difficult to tell which stars are which on these diagrams, we have plotted certain stars as open circles on all three figures. These stars are the long-period group in Figures 2 and 14, i.e., those stars longward of ten

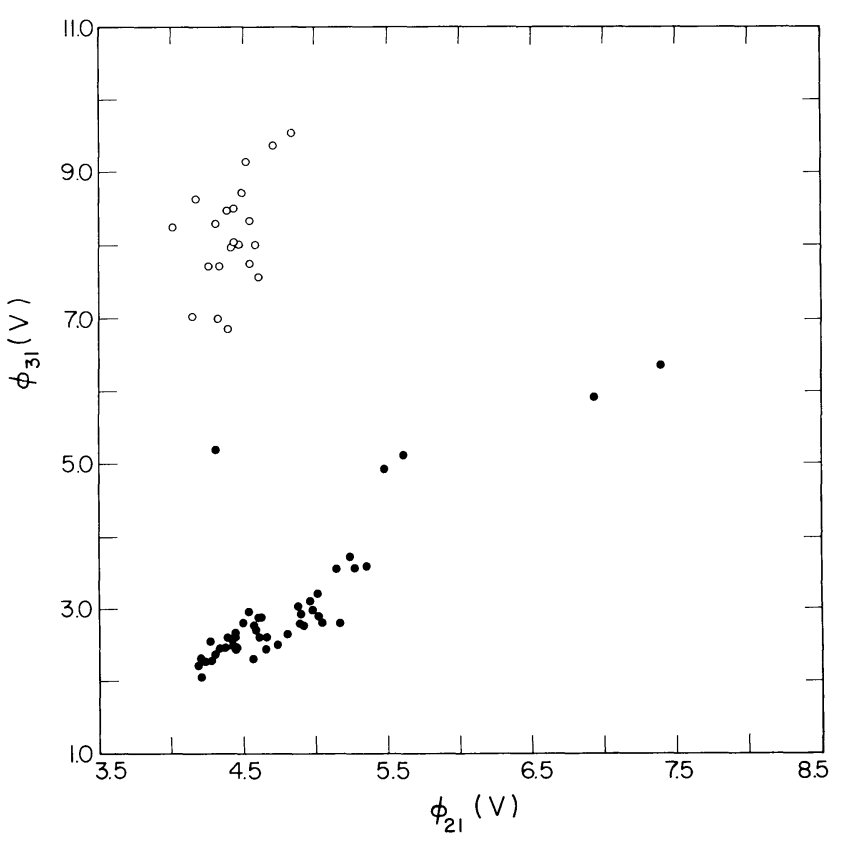

FIG. 15-The phase difference $\phi_{31}$ versus $\phi_{21}$ for the $V$-magnitude data. The open circles are the long-period stars in Figures 2 and 14.

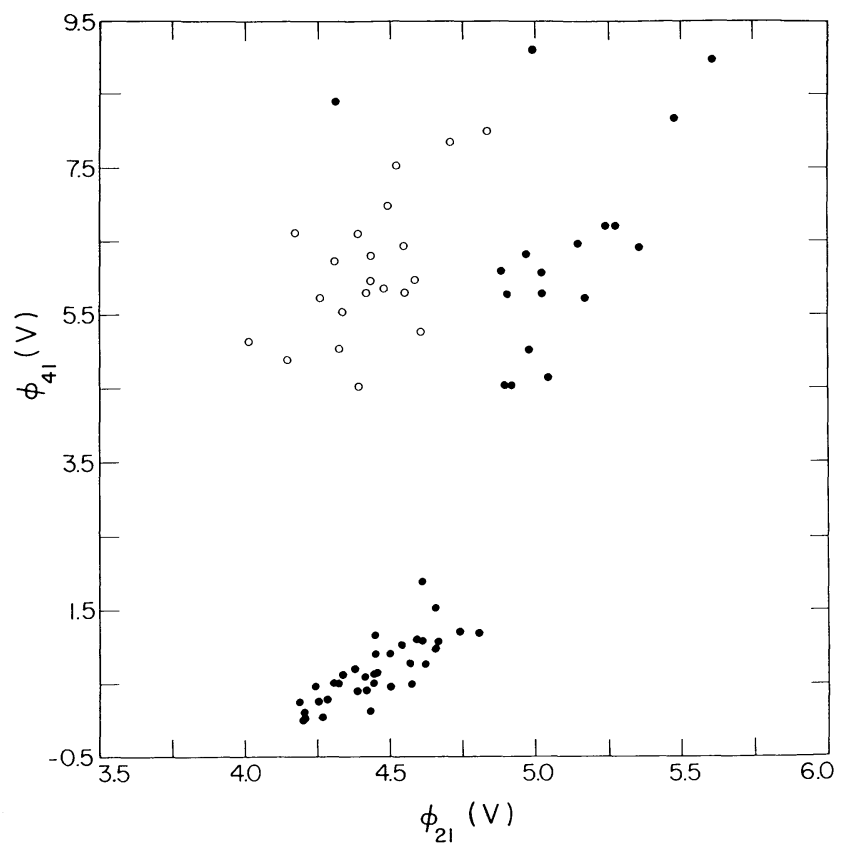

FIG. 16-The phase differences $\phi_{41}$ versus $\phi_{21}$ for the $V$-magnitude data. The open circles are long-period stars. days whose values of $\phi_{21}$ and $\phi_{41}$ have dropped off sharply from the resonant peak.

We note that the resonance progression among the shorter-period stars ( $P \leq 10$ days) appears clearly in all three of the phase-phase plots. However, it is also clear that a discontinuity exists between these objects and the longer-period stars, particularly in Figures 15 and 17. This is interesting because no such discontinuity is apparent in the $\phi_{31}$-period plot (Fig. 4), and even in the $\phi_{21}$ and $\phi_{41}$ vs. period diagrams (Figs. 2 and 14) if one eliminates the handful of points close to ten days the argument for a discontinuity becomes much weaker.

In our opinion, Figures 15-17 constitute evidence in favor of the idea that the Cepheids with periods above ten days are somehow "different" from those with periods below ten days. While Davis, Moffett and Barnes (1981) have suggested that the difference may actually be one of mass, this idea is hard to reconcile with the theory of stellar evolution. A more promising suggestion is that made recently by Klapp, Goupil, and Buchler (1985). On the basis of comparisons between nonlinear perturbation models, on the one hand, and hydrodynamic calculations and observational data, on the other, these authors have proposed that long-period and short-period Cepheids may have "a different attractor or saturation mechanism." This proposal needs to be explored further.

Finally, it should be kept in mind that any of the Fourier phases may, of course, be altered in a given plot by an amount $\pm 2 \pi n$ ( $n$ an integer). The values we have chosen are those which seemed most consistent with the requirements that the diagrams be compact and, where

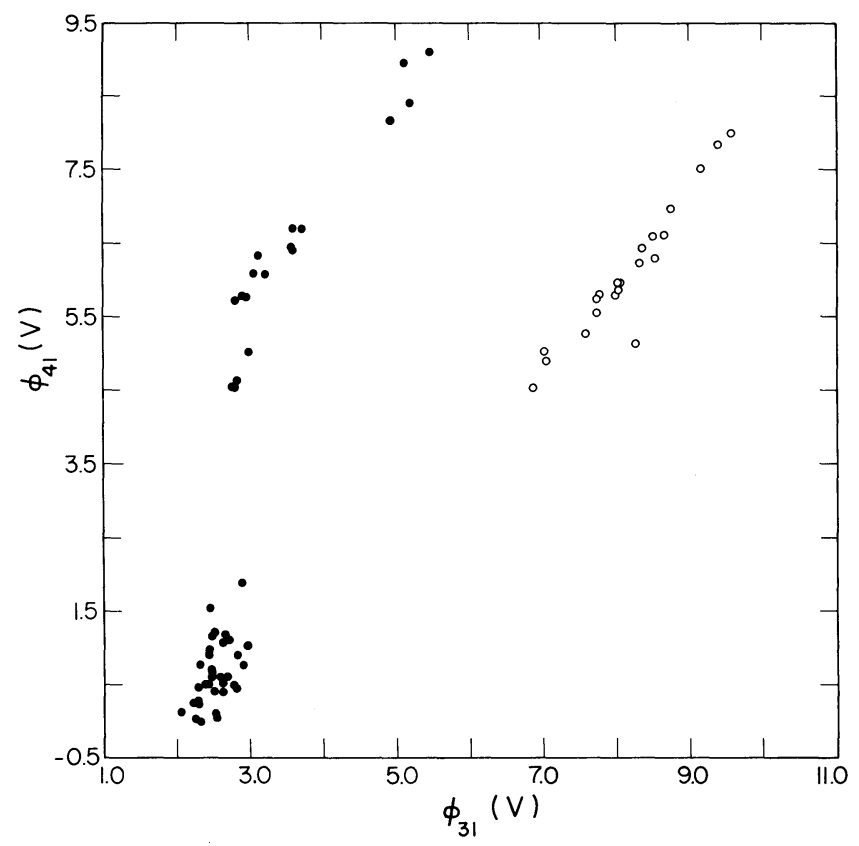

FIG. 17-The phase difference $\phi_{41}$ versus $\phi_{31}$ for the $V$-magnitude data. The open circles are long-period stars. 
possible, continuous. However, other choices are possible. For example, in Figure 15 one could subtract $2 \pi$ from each of the open circles, moving these points down to coincide with the dots below. The same change would move the open circles somewhat closer to the dots in Figure 17. However, in Figure 4 this alteration introduces a discontinuity at about 6.5 days in a plot that otherwise looks remarkably continuous. There seems no good reason for forcing such a disruption. Similarly, one might consider subtracting $2 \pi$ from the four highest points in Figures 14 and 17. These points could then be interpreted as lying on the low end of the long-period sequence (open circles in Fig. 17). On the other hand, this change does not work so well in Figure 16, particularly as concerns the two points at the upper right. Because of these uncertainties, the present form of our phase-phase diagrams should not be taken as final, but rather as a first attempt at meaningfully displaying the points. It is likely that definitive plots will have to await a better understanding of the physical and mathematical effects which underlie the Fourier phases.

We are pleased to acknowledge support from the $\mathrm{Na}$ - tional Science Foundation under grants AST 83-16875 (N.R.S.) and AST 84-17844 (T.J.M.).

\section{REFERENCES}

Antonello, E., and Mantegazza, L. 1984, Astr. Ap. 133, 52.

Arellano-Ferro, A. 1984, M.N.R.A.S. 209, 481.

Davis, C. G., Moffett, T. J., and Barnes, T. G. 1981, Ap. J. 246, 914.

Gieren, W. 1981, Ap. J. Suppl. 47, 315.

Gieren, W. 1982, Pub. A.S.P. 94, 360.

Hodson, S. W., Cox, A. N., and King, D. S. 1982, Ap. J. 253, 260.

Klapp, J., Goupil, M. J., and Buchler, J. R. 1985, Ap. J. (in press).

Moffett, T. J., and Barnes, T. G. 1980, Ap. J. Suppl. 44, 427.

Moffett, T. J. and Barnes, T. G. 1984, Ap. J. Suppl. 55, 389.

Moffett, T. J. and Barnes, T. G. 1985, Ap. J. Suppl. 58, 843.

Payne-Gaposchkin, C. 1947, A.J. 52, 218.

Petersen, J. O. 1984, Astr. Ap. 139, 496.

Petersen, J. O., and Hansen, L. 1984, Astr. Ap. 134, 319.

Schmidt, E. G. 1985 (private communication).

Simon, N. R. 1984a Ap. J. 284, 278.

Simon, N. R. 1984b, Bull. A.A.S. 1984, 16, 970.

Simon, N. R., and Davis, C. G. 1983, Ap. J. 266, 787.

Simon, N. R., and Lee, A. S. 1981, Ap. J. 248, 291.

Simon, N. R., and Teays, T. J. 1982, Ap. J. 261, 586.

Simon, N. R., and Teays, T. J. 1983, Ap. J. 265, 997.

Teays, T. J., and Simon, N. R. 1982, in Pulsations in Classical and Cataclysmic Variables, J. P. Cox and C. J. Hansen, eds. (Boulder: JILA), p. 242. 